\title{
Reflexiones Conceptuales DE LA ADAPTACIÓN BIOLÓGICA Y SU RELACIÓN CON EL EJERCICIO FÍSICO
}

* FT. Jorge Enrique Correa Bautista

\section{Resumen}

El presente artículo aborda la teoría de adaptación biológica y su relación con el ejercicio en todos sus niveles; relación que se ve reflejada en los cambios cardiovasculares, pulmonares, renales osteomusculares, inmunológicos y hematológicos frente a una situación de ejercicio físico, los cuales explicarán la funcionalidad del cuerpo humano ante un estrés fisiológico generado por el entorno.

Palabras Clave: Adaptación biológica, ejercicio físico

$\mathrm{T}$ odo ser vivo interactúa permanentemente dentro de un medio, por lo cual su organismo reacciona a cada estímulo que el medio le brinda; estas reacciones involucran cada célula que lo conforma y cada una de ellas a su vez responde ante el estímulo; por ende, los tejidos, los órganos y los sistemas que están formados por miles de células, también reaccionarán de diferente manera frente a cada estímulo. Esta capacidad de respuesta se constituye en una de las propiedades fundamentales para la adaptación de cualquier especie.

Los estímulos del medio por lo general son sensoriales y recibidos en el organismo por medio de los receptores, los cuales producen una respuesta dependiendo del receptor que es excitado. El estímulo es captado y se produce un potencial de acción que se traduce en una despolarización de la membrana celular por intercambio iónico; este potencial de acción desciende con el paso del tiempo cuando el estímulo sostenido de intensidad constante se aplica sobre un receptor, produciendo el fenómeno de adaptación. Este concepto neurológico de adaptación es el mismo mecanismo de excitación-reacción, el cual fue expuesto por Hans Seluy (1960) como el "síndrome general de adaptación” y refiere que

* Fisioterapeuta del Colegio Mayor de Nuestra Señora del Rosario. Especialista en Gerencia de las Organizaciones de Salud. Especialista en docencia Universitaria. Docente de la Escuela Colombiana de Rehabilitación. Director de la línea de investigación ejercicio fisico de la Facultad de Rehabilitación y Desarrollo Humano de la Universidad del Rosario 
ante un estímulo excitatorio el organismo internamente responde y realiza cambios para no quedar en imbalance.

Esta concepción neurológica nos indica que el organismo del hombre reacciona con cualquier estímulo y pasado un tiempo en el cual el estímulo se realiza de forma constante, su reacción disminuye, haciendo que se produzca una adaptación hacia el estímulo; esto es lo que sucede cuando el estímulo o el ejercicio físico es realizado de forma frecuente, generando procesos de adaptación que se explicarán a lo largo de este documento.

No sólo las reacciones ante un estímulo permiten al ser vivo su adaptación, existen otras propiedades como la capacidad de convertir energía química en energía mecánica y la capacidad de movimiento, que permiten rápidos ajustes fisiológicos lo que garantiza suplir las necesidades básicas.

Estas propiedades sumadas a la capacidad de reproducción, aseguran la supervivencia de cada especie, como lo explica Darwin (1859), haciendo referencia al concepto de "lucha por la vida", donde la victoria ha de corresponder siempre a quien posea una ventaja respecto a sus competidores. Estas ventajas distinguen sólo a algunos individuos y pueden parecer de poca importancia hasta el momento en que al ser necesarias determinan la selección natural y la supervivencia de los más aptos. El resultado es un proceso continuo de adaptación, así como la aparición de mejoras dentro de los individuos de una especie.

Darwin manifestó un interés por las adaptaciones que realizan los animales para lograr vivir en su medio, sus teorias han correlacionado tres conceptos explicando el éxito de la supervivencia; como primer concepto, se tiene el de la adaptación biológica, el cual enuncia que la adaptación es el "estado de encontrarse ajustado al medio" como una característica particular del ser vivo; el segundo concepto tiene que ver con la adaptación fisiológica o fenotípica, la cual se relaciona con los cambios que se generan en un individuo frente a un estímulo particular, y por último el concepto evolutivo o de adaptación genotípica, el cual considera la adaptación como un proceso que ocurre dentro de toda una población durante el curso de muchas generaciones.

Estas tres concepciones se complementan para entender y explicar los procesos de adaptación que realiza un organismo para su supervivencia a través de su espacio y tiempo de vida. Así mismo, estos niveles de adaptación se aplican al hombre, por ejemplo un buceador cuando reacciona al cambio de presión o un astronauta cuando pasa de la gravitación a la ingravidez, hacen referencia al concepto de adaptación biológica.

El ejercicio físico, entendido como un conjunto de actos motores que agrupados bajo una intencionalidad en particular favorecen el incremento de la capacidad cinética o de desempeño de un individuo (Línea de investigación en ejercicio físico Universidad del Rosario, 2000) potencializan su resistencia y mejoran su capacidad de adaptación en todos los niveles.

De allí, que abordar la práctica de ejercicio físico, como un estimulo externo generador de una sobrecarga en el organismo que se encuentra en movimiento obliga a reflexionar acerca de la importancia y el papel del mismo como elemento fundamental para la supervivencia y el bienestar de la especie.

Desde la época de los griegos y los romanos, la sociedad reconocía en el ejercicio físico un potencializador no sólo de las cualidades físicas sino también de las cualidades morales; conocían también la relación estímulo-respuesta-adaptación porque observaban lo que pasaba cuando un individuo practicaba con frecuencia ejercicio físico: cambios en el cuerpo como aumento de la fuerza, de la potencia y de la velocidad y con el paso del tiempo se obtenía una mayor capacidad de resistencia. Esto nos demuestra que el interés frente a la adaptación no es nuevo.

Lamarck (1890) retomó la idea de los griegos acerca del ejercicio físico y planteó el efecto que tenía éste sobre un organismo, explicando que la utilización de cualquier órgano lo fortalece y lo hace crecer y desarrollar, mientras que si no es utilizado se debilitará, disminuyendo sus capacidades hasta su desaparición. 


\section{ARTÍCULOS INÉDITOS}

Este concepto reafirma que la realización del ejercicio físico de forma frecuente mejora y potencializa las cualidades físicas del organismo y cuando sucede esto el cuerpo realiza adaptaciones que le permiten su autodesarrollo .

El mejoramiento de la condición física por medio del ejercicio físico es reconocido por Monogarov, Vladimir Dmitriyevich Mishenko, Victor Sergeyevich,(1991) cuando definieron el proceso de adaptación como la consideración del dinamismo de crecimiento de la capacidad del trabajo físico, concepto en el cual está implícito que el crecimiento del individuo depende del trabajo dinámico que este realice.

Por tanto, los procesos de adaptación están relacionados directamente con la frecuencia de la realización del ejercicio físico, es decir, que a mayor ejercicio mayores son las adaptaciones que el cuerpo realiza. Estos cambios involucran un estrés o esfuerzo que estimula los procesos de adaptación fisiológica y biológica, principalmente.

En este sentido, se entiende por estrés ( Hans Selye. 1940) como "la respuesta no específica del organismo a cualquier demanda del exterior". Ante un estrés como el esfuerzo o la ejercitación, el organismo se debe enfrentar a distintos estímulos, por ejemplo, el número de carga combinado con intensidad y duración y con movimientos de coordinación que se efectuarán con los distintos ejercicios encaminados a mejorar las cualidades físicas y mentales.

Autores como Meerson, Kutnesov y Verjoshanski (1970) definieron la adaptación como el "proceso a través del cual el hombre se adecua a las condiciones naturales de vida, de trabajo, y en general a cualquier actividad, llevando a una mejora morfológica-funcional del organismo y a un aumento de su potencialidad vital y de su capacidad no específica de resistir a los estímulos extremos del ambiente. En este concepto cabe destacar que los autores además de plantear que la potencialidad vital aumenta mediante la realización de un proceso de ejercitación ,mencionan también que existe una capacidad no identificada que puede ser llamadas reserva funcional, la cual permite al organismo sobrevivir ante estímulos extremos. Con este concepto se puede plantear los efectos de un proceso de acondicionamiento fisco sobre la potencialidad vital en los individuos.

Platonov (1991) proporcionó una definición de la teoría de la adaptación biológica desde el punto de vista anatómico y fisiológico. En 1994, el mismo autor en su libro La Adaptación en el Deporte, planteó el concepto de adaptación biológica como proceso y como un resultado: proceso porque implica un tiempo y etapas para acostumbrarse a los factores internos y externos y resultado porque es la consecuencia o el final de los prócesos, por lo tanto involucra un desarrollo, unos mecanismos, unas leyes y unas direcciones de las reacciones. Estas dos definiciones son válidas para referirse a la adaptación porque esta sucede cuando transcurre determinado tiempo y trae consecuencias favorables al organismo.

Aparte de los conceptos morfofisiológicos que describen la adaptación biológica, surgieron varios autores que involucran no solo la dimensión fisiológica del ser, sino su parte conductual. P. Jochachka y D. Somero (1965) expusieron que existen tres rutas por las cuales se puede conseguir la adaptación biológica en un individuo. La primera de ellas la fisiológica, la cual promulga la producción de cambios en la función de órganos y sistemas, la segunda es la bioquímica la cual actuará si se agotan las reservas y es imprescindible como reguladora de todas las funciones de micro y macromolécula, y la tercera ruta, la conductual, la cual hace referencia a un componente psicológico y mental dentro de los procesos de adaptación biológica. Esta última ruta es un elemento innovador para entender los procesos de adaptación, ya que involucra la dimensión psicológica, la cual es parte fundamental de la esencia misma del ser.

Otros dos autores que mencionaron la conducta dentro de la adaptación fueron P. Anojín (1980) y K. Sudakóv (1983) quienes consideraron que las adaptaciones biológicas que realiza el organismo durante el ejercicio están íntimamente relacionadas con la motivación y la conducta del hombre hacia un objetivo 
determinado, con lo cual empieza a exponer que la parte emocional y psicológica del organismo influencian la ejecución del ejercicio físico de una manera directa.

Como se ve, casi todos los conceptos expuestos por diversos autores, basan su teoría en el "concepto Darwinista", ya que en cada definición está expuesta la idea de adaptación en relación con el convivir y con el medio, el cual provee de diversos estímulos al organismo humano haciendo que éste produzca respuestas sobre el estímulo impuesto; por lo tanto, puede quedar muy superficial el concepto si se liga solo a respuestas fisiológicas. Las adaptaciones van más allá que simples respuestas, son mecanismos que favorecen el funcionamiento del ser humano y hacen que éste realice todas sus tareas de una forma más fácil, con lo cual el organismo humano contará con mayor resistencia para realizar su actividad física. Por esto es importante tener en cuenta la concepción de Platonov, ya que no sólo describió el modo fisiológico como suceden las adaptaciones biológicas sino que las toma como un proceso y como un resultado; además, sus estudios de adaptación los basa en el entrenamiento físico el cual se puede considerar como un elemento esencial y al ejercicio físico como una herramienta de prevención contra enfermedades y una estrategia para lograr un bienestar total, objetivo principal que busca la sociedad moderna mediante la ejercitación.

Es importante tener en cuenta que el estado psicológico y emocional están unidos estrechamente a la ejercitación y por ende influirán en los procesos de adaptación que ocurren con el entrenamiento, por lo tanto el concepto conductual expuesto por los autores Anojín, Sudakóv, Jochachka y Somero son válidos en este trabajo sólo como factor que interviene en la adaptación biológica, junto con otros factores como la individualización del ejercicio, ya que cada persona presenta diferentes características físicas y respuestas al entrenamiento físico. Otro factor que encontramos es el estado físico de cada persona ya que es el responsable del tiempo y el esfuerzo para conseguir adaptaciones biológicas. Por último, tenemos como factor la potencia de cada individuo la cual dependerá del estado físico y de su material genético.

El hecho de tomar la adaptación biológica como un proceso y como un resultado implica al profesional que prescriba el ejercicio tener en cuenta dicha relación, con el fin de generar cambios que beneficien la capacidad vital y de movimiento de los individuos que deciden ejercitarse.

\section{Referencias Bibliográficas}

Åstrand, P-O., \& Rodahl, K. (1986). Textbook of Work Physiology: Physiological Bases of Exercise. (3rd ed.). New York: McGraw-Hill Book Company.

Åstrand, P-O., \& Rodahl, K. (1986). Fisiología del Trabajo Físico: Bases Fisiológicas del Ejercicio. (2da ed.). Buenos Aires, Argentina: Editorial Médica Panamericana S.A.

Brooks, G. A., \& Fahey, T. D. (1987). Fundamentals of Human Performance. New York: Macmillan Publishing Company.

Books, G. A., Fahey, T. D., \& White, T. P. (1996). Exercise Physiology: Human Bioenergetics and its Applications. (2ndh ed.). California: Mayfield Publishing Company. 750 pp.

De Vries, H. A., \& Housh, T. J. (1994).

Fox's Physiological Basis for Exercise and Sport. (6th ed.) Boston: WCB/McGraw-Hill Company, Inc. 620 pp.

Fox, E. L., Bowers, R. W., \& Foss, M. L. (1992). Sports Physiology. (3rd ed). Wisconsin: WCB Brown \& Benchmark Publishers. 449 pp.

Fox, E. L. (1987). Fisiología del Deporte. (5ta ed., Reimpresión de la 1 ra ed.). Buenos Aires, Argentina: Editorial Médica Panamericana. $351 \mathrm{pp}$.

Gonzalez Gallego, J. (Ed.). (1992). Fisiología de la Actividad Física y del Deporte. Nueva York: Interamericana, McGrawHill.

Lamb, D. R. ,\& Murray, R. (Eds.) (1997). Lamb, D. R. (1984). Physiology of Exercise: Responses \& Adaptations. (2nd. ed.). New York: Macmillan Publishing Company.

McArdle, W. D., Katch, F. I., \& Katch, V. L. (1996). Exercise Physiology: Energy, Nutrition, and Human Performance. (4th ed.). Baltimore, Maryland: Williams \& Wilkins. 849 pp.

Mishchenko, V. S., \& Monogarov, V. D. (1995). Fisiología del Deportista: Bases Científicas de la Preparación, Fatiga y Recuperación de los Sistemas Funcionales del Organismo de los Deportistas de Alto Nivel. Barcelona, España: Editorial Paidotribo.

Morehouse, L. E., \& Miller, A. T., Jr. (1984). Fisiología del Ejercicio. (8va ed.). Buenos Aires: Librería "El Ateneo" Editorial.

Noble, B. J. (1986). Physiology of Exercise and Sport. St. Louis: Times Mirror/Mosby College Publishing. 


\section{ARTICULOS INÉDITOS}

Plowman, S. D., \& Smith, D. L. (1997). Exercise Physiology for Health, Fitness, and Performance. Boston: Allyn and Bacon. $557 \mathrm{pp}$.

Reilly, T., Secher, N., Snell, O., \& Williams, C. (Eds.). (1990). Physiology of Sports. New York: E. \& F.N. SPON. 495 pp.

Powers, S. K., \& Howley, E T. (1996). Exercise Physiology: Theory and Applications to Performanve. (3rd ed.). Boston: WCM/McGraw-Hill Companies. 522 pp.

Robergs, R. A., \& Roberts, S. O. (2000). Fundamental Principles of Exercise

Physiology: For Fitness, Performance, and Health. Boston: McGraw Hill Companies. 487 pp.

Robergs, R. A., \& Roberts, S. O. (1997). Exercise Physiology: Exercise, Performance, and Clinical Applications. St. Louis: Mosby-Year Book, Inc. 840 pp.

Rowell, L. B., \& Shepherd, J. T. (Eds). (1996). Handbook of Physiology. A Critical, Comprehensive Presentation of Physiological Knowledge and Concepts. Section 12: Exercise: Regulation and Integration of Multiple Systems. New York: Oxford University Press.

Stegeman, J. (1981). Exercise Physiology: Physiological Bases of Work and Sport. St. Louis: Year Book Medical Publishers

Wilmore, J. H., \& Costill, D. L. (1994). Physiology of Sport and Exercise. Champaign, IL: Human Kinetics.

Wilmore, J. H., \& Costill, D. L. (1988). Training for Sport and Activity. (3rd ed.). Madison, WI: Wm. C. Brown Publishers. 